\title{
Company Product Development
}

\begin{tabular}{ll}
\hline Baxter Healthcare Factor VIII & The European Community's (EC) Committee for Proprietary Medical Products has \\
recommended the approval of Factor VIII to treat hemophilia A. This sets the stage
\end{tabular} for EC member countries to approve the product.

Biocine gD2 vaccine Results from phase II studies show that 98 patients with genital herpes who were vaccinated at 0 and 2 months with the gD2 immunotherapeutic experienced one-third fewer outbreaks than the control group.

BioCryst $\quad B C X-34 \quad B C X-34$, an inhibitor of the enzyme purine nucleoside phosphorylase, showed efficacy in treating both cutaneous T-cell lymphoma and psoriasis in phase I/II trials.

Cell Therapeutics CT-1501R

CT-1501R has commenced four clinical studies as a therapy to lessen the toxicities of chemotherapy and interleukin-2 therapies in cancer patients.

Centocor Myoscint \& Fibriscint

Centocor has withdrawn the product license applications (PLA) for Myoscint and Fibriscint following the discovery of deficiencies in the applications. The PLA for Myoscint, a monoclonal antibody (MAb) based cardiac imaging agent, was filed in 1988. The PLA for Fibriscint, a MAb-based blood-clot imaging agent, was filed in 1991.

Cor Therapeutics Integrelin

Phase II results of Integrelin, a glycoprotein IIb-IIla antagonist, indicate that it reduces clinical events when administered with aspirin and heparin as treatment for angioplasty.

Immunogen Oncolysin B Phase I results for Oncolysin B show that the immunoconjugate extended the remissions of 10 of $12 \mathrm{~B}$-cell lymphoma patients. The patients were in remission subsequent to autologous bone-marrow transplants.

Liposome Doxil

Technology

Doxil, liposome-encapsulated doxorubicin hydrochloride, delivered five-to-eleven times more drug than comparable doses of Adriamycin, unencapsulated doxorubicin, to AIDS patients with Karposi's sarcoma.

Matrix MPI 5003

MPI 5003, an injectable therapeutic implant containing fluorouracil, will start phase III trials against genital warts. Phase II results showed that 65 percent of patients treated with the product had complete clearance of genital warts.

Matrix $\quad$ MPI 5011

Early results from ongoing phase I/II trials of MPI 5011 are encouraging. The product is an injectable therapeutic implant for prostate cancer that contains 5-fluorouracil.

Medarex MDX-210 Medarex is beginning phase $\mathrm{I} / \mathrm{II}$ trials of a bispecific antibody, MDX-210, for breast and ovarian cancers.

Neoprobe

RIGScan CR49 Neoprobe will commence a second phase III trial for its RIGScan CR49, the radiolabeled-antibody component of its intraoperative cancer-detection system. The trial will evaluate patients diagnosed with metastic colorectal cancer.

NeoRx OncoTrac NeoRx has commenced a limited clinical trial to qualify Boehringer Ingelheim as the manufacturer of its OncoTrac small-cell lung-cancer imaging kit.

Protein Antibodies

Design Labs

The Food and Drug Administration (FDA) has granted orphan drug status for certain indications to Protein Design Lab's human antibodies against cytomegalovirus and hepatitis B.

Ribi ImmunoChem MPL-C

FDA has allowed an investigational new drug application to evaluate the prophylactic use of MPL-C immunomodulator to reduce the effects of cardiac ischemia-reperfusion injury. MPL-C is thought to increase production of a protective antioxidant enzyme in cardiac cells.

Sandoz

Interleukin-3

Sandoz has begun a phase III trial for combination therapy with interleukin-3 and granulocyte macrophage colony stimulating factor. The trial aims to reconstitute blood-cell populations following autologous bone-marrow transplantation in patients with Hodgkin's disease or non-Hodgkin's lymphoma.

Seragen $\quad \mathrm{DAB}_{389} \mathrm{IL}-2$ Seragen has begun a phase $\mathrm{V} / \mathrm{II}$ trial for early treatment of $\mathrm{HIV}$ with $\mathrm{DAB} \mathrm{B}_{389} \mathrm{IL}-2$, the second version of its interleukin-2 receptor-targeted fusion toxin.

Seragen $\quad \mathrm{DAB}_{486} \mathrm{IL}-2$

In a phase II trial, some patients with refractory rheumatoid arthritis showed clinical improvement after receiving three courses of $D A B_{486} \mathrm{~L}-2$, Seragen's initial interleukin-2 receptor-targeted fusion toxin.

Sphinx Kynac Preliminary results of a phase II trial of Kynac ointment for treatment of psoriasis Pharmaceuticals Kynawed no statistically significant difference between Kynac and its ointment vehicle. Kynac inhibits the activity of the protein kinase $C$ family of intracellular enzymes. 